\title{
School Choice: An analysis of the strategic behaviour of students and schools in the student high-school allocation problem
}

\author{
Kyara Lucas*
}

\begin{abstract}
We investigate how the one-to-one deferred acceptance algorithm can be used to solve the many-to-one school choice problem. In the first part of the paper we derive the important properties of the one-to-one Deferred Acceptance algorithm. In the second part of the paper, we consider the Deferred Acceptance algorithm for solving the many-to-one school choice problem. Using insights from the first two parts of the paper we show that the Deferred Acceptance algorithm is strategy proof for students, and manipulable by schools under specific conditions.
\end{abstract}

\section{Introduction}

\subsection{Problem Background}

In Maastricht, some 1,000 children transfer from primary school to secondary school each year. Problems arise when parents are not happy with the allocation of their children and an after-market is formed for re-allocation of students to high schools. This is a typical example of unstable allocations; such market design gives rise to frustration and provokes strategic behavior.

\subsection{Purpose and Research Question}

This paper sets out to research the implementation of the Gale-Shapley Deferred Acceptance Algorithm to assign students to their preferred school choice. The Deferred Acceptance algorithm is widely used for solving one-to-one matching problems, we will examine to what extent the properties of this matching algorithm are preserved when dealing with a many-to-one case. An important issue being whether agents have an incentive to tell the truth or whether they can manipulate results by misreporting their

${ }^{*}$ Kyara Lucas will receive a bachelor degree in Econometrics \& Operations Research at Maastricht University in 2018. She recently started working as a data scientist at Atos.

Contact: kyaralucas@live.com

School Choice: An analysis of the strategic behaviour of students and schools in the student high-school allocation problem 
preferences. Whilst there has been a lot of previous research on the strategic behavior of students, less is known on the strategic behavior of schools in this setting. The purpose of this paper is to extend the deferred acceptance algorithm for many-to-one matching problems, and to assess the strategic behaviour of both students and high-schools in this model. Specifically, this analysis will be concerning the following questions:

- How can we characterize the strategic behavior of both students and schools?

- To what extend is truthful reporting a dominant strategy?

- Is manipulation possible for schools in the many-to-one case, if so what are the conditions for being able to do so?

We will try to answer these questions by showing that the one-to-one properties of the Deferred Acceptance algorithm also hold in the many-to-one case. Furthermore, we try to find results by analyzing the effect of one student or school misreporting their preference to see to what extent they are able to improve their own matching. We consider cases with three schools with two different preferences and four students and check for manipulation. Using these approaches, we aim to expand on previous literature regarding the Deferred Acceptance algorithm for solving the school choice problem.

\subsection{Thesis Disposition}

This paper proceeds as follows. The next section covers another school choice allocation mechanism with its limitations. Section 3 presents and discusses the properties of the Deferred Acceptance algorithm in the one-to-one case. Section 4 extends the mechanism and provides details of the properties of the many-to-one case. Furthermore, section 4 presents and discusses the strategic behavior of schools whilst utilizing the many-to-one deferred acceptance algorithm. Finally, section 5 summarizes and concludes our findings.

\section{The Boston School Choice Mechanism}

While the aim of this paper is to assess the strategic behavior of both students and schools in the Deferred Acceptance algorithm, it is important to also consider other allocation mechanisms and their shortcomings. A widely-used student school allocation algorithm is the Boston School Choice Mechanism. The algorithm works such that spots of each school are allocated to students who have the school as first in their preferences, then to those who have the schools as the second if there are any spots left, and so forth. Thus, under the mechanism, each school allocates spots to all qualified students who rank it higher before allocating to students who rank it lower. Whilst the Boston School Choice mechanism is a very popular student-placement mechanism, it has various shortcomings. Abdulkadiroglu and Sonmez (2003) show that the mechanism may result in unstable matches. Furthermore, the Boston School Choice mechanism is not strategy-proof and provokes manipulation [2].

$\mathbf{8 0} \mid \begin{aligned} & \text { Marble } \\ & \text { Research } \\ & \text { Papers }\end{aligned}$




\section{The Deferred Acceptance Algorithm}

In this section the Deferred Acceptance Algorithm will be presented. Firstly, we will introduce the stable marriage problem. Following that, we will prove the most important properties of the Deferred Acceptance one-to-one matching.

\subsection{The Stable Marriage Problem}

The Deferred Acceptance Algorithm proposed by Gale and Shapley is an approach to solving the stable marriage problem (SMP). The stable marriage problem is the problem of finding a one-to-one matching between elements of the two sets of $\mathrm{n}$ women and $\mathrm{n}$ men, where each person provides a complete strict ranking/preference list of the members of the opposite set. A matching is a set of pairs $(\mathrm{m}, \mathrm{w})$ such that each individual has one partner. A matching is considered stable when there's no $\mathrm{m}$ or w who would both rather be matched to each other rather than their current partners. A matching is considered unstable if there exists a pair m-w who would prefer to be matched together than their current partners, such a pair is referred to as a blocking pair. Firstly, we define the following:

- Set of men $M=\left[m_{1}, \ldots, m_{n}\right]$

- Set of women $W=\left[w_{1}, \ldots, w_{n}\right]$

- Set of preferences $\mathrm{P}$ including preferences of each $\mathrm{m}$ in $\mathrm{M}$ and $\mathrm{w}$ in $\mathrm{W}$

- Matching S consisting of n pairs $(m, w)$

- One-to-one matching: each $\mathrm{m}$ can only be matched to one w, and vice-versa

The algorithm has two versions namely the man-proposing version and the woman proposing version. The man-proposing version works as follows:

Data: $(\mathrm{M}, \mathrm{W}, \mathrm{P})$

Result: stable matching $\mu$ initialization;

while not all $m$ in $M$ are matched do

choose $\mathrm{m}$ (who is not yet matched) to propose to their most preferable w (who has not yet rejected them);

if this is the first proposal $w$ receives $\vee w$ values $m$ more than her current match then

I $\mathrm{w}$ accepts $\mathrm{m}$, match $\mathrm{w}$ and $\mathrm{m}$

else

w rejects $\mathrm{m}$;

end

end

Algorithm 1: Men proposing Deferred Acceptance algorithm

Since all preference profiles in $\mathrm{P}$ are reported with strict inequalities, it is important to note that the order in which all $\mathrm{m}$ in $\mathrm{M}$ propose does not matter. Furthermore, for the remainder of this paper we will focus on the version of the Deferred Acceptance

School Choice: An analysis of the strategic behaviour of students and schools in the student high-school allocation problem 
algorithm where men propose. To fully grasp how the algorithm operates, we construct a $3 \times 3$ example.

Example 3.1. We are given the following preference profiles (Table 1 and table 2). Note that Table 1 states that $m_{1}$ prefers $w_{1}$ over $w_{2}$ over $w_{3}$.

\begin{tabular}{|l|lll|}
\hline$m_{1}$ & $w_{1}$ & $w_{2}$ & $w_{3}$ \\
$m_{2}$ & $w_{3}$ & $w_{2}$ & $w_{1}$ \\
$m_{3}$ & $w_{3}$ & $w_{1}$ & $w_{2}$ \\
\hline
\end{tabular}

Table 1: Male preferences

\begin{tabular}{|l|lll|}
\hline$w_{1}$ & $m_{2}$ & $m_{3}$ & $m_{1}$ \\
$w_{2}$ & $m_{1}$ & $m_{2}$ & $m_{3}$ \\
$w_{3}$ & $m_{1}$ & $m_{3}$ & $m_{2}$ \\
\hline
\end{tabular}

Table 2: Female preferences

In the first round of the algorithm $m_{1}$ proposes to $w_{1}$, as this is the first proposal $w_{1}$ receives, she accepts, which results in the pair $\left(m_{1}, w_{1}\right)$. Next $m_{2}$ proposes to $w_{3}$, same holds thus resulting in the pair $\left(m_{2}, w_{3}\right)$. Finally, $m_{3}$ proposes to $w_{3}$, as $w_{3}$ prefers $m_{3}$ over $m_{2}$ she accepts the proposal resulting in the pair $\left(m_{3}, w_{3}\right)$. Consequently $m_{2}$ is unmatched. The first round of the algorithm terminates with only one $m$ in $M$ remaining unmatched. Thus, in the second round of the algorithm $m_{2}$ proposes to the next woman in his preferences which is $w_{2}$, as this is the first proposal $w_{2}$ receives, she accepts. Thus, the algorithm terminates at round $n=2$ with the matching $\left[\left(m_{1}, w_{1}\right) ;\left(m_{2}, w_{2}\right) ;\left(m_{3}, w_{3}\right)\right]$.

\subsection{Properties}

The deferred acceptance algorithm is widely implemented for it's useful properties. Firstly, each matching is perfect thus each $\mathrm{m}$ in $\mathrm{M}$ is matched to a $\mathrm{w}$ in $\mathrm{W}$ and vice versa. Secondly, each matching $U$ is stable thus there exists no pair that would rather be matched together than their current match. The following two lemmas follow directly from the algorithm's structure:

Lemma 3.1. All $m$ in $M$ propose to $w$ in $W$ in a decreasing order of preference.

Lemma 3.2. Once a $w$ in $W$ is matched, she is never unmatched; she can only improve by trading up.

By lemma 3.2 we will show that the resulting Deferred Acceptance matching is complete i.e. all men and woman have a match.

Theorem 3.3. Upon termination of the algorithm all men and women are matched.

Proof. This statement will be proven with a proof by contradiction. Assume that the algorithm finishes and there exists some $m_{1}$ who is not matched to any $\mathrm{w}$ in $\mathrm{W}$. Then, as $|W|=|M|$ we know that there exists some woman $w_{1}$ who is also not matched. By Lemma 3.2 we may conclude that $w_{1}$ was never proposed to but we know that $m_{1}$ proposes to all $\mathrm{w}$ in $\mathrm{W}$ since he ended up unmatched. This is a contradiction.

$82 \mid \begin{aligned} & \text { Marble } \\ & \text { Research } \\ & \text { Papers }\end{aligned}$ 
By lemma 3.1 we will show that the resulting Deferred Acceptance matching is stable i.e. there are no blocking pairs.

Theorem 3.4. Upon termination of the algorithm all pairs in $S$ are stable.

Proof. Assume there exists a pair $\left(m_{1}, w_{1}\right)$ which is a blocking pair, thus $m_{1}$ and $w_{1}$ prefer to be matched together than to their current match according to the DA matching. We distinguish between two cases:

Case 1: $m_{1}$ never proposed to $w_{1}$.

By Lemma 3.1. we know that this can only occur if $m_{1}$ prefers his current DA matching partner to $w_{1}$. This is a contradiction.

Case 2: $m_{1}$ proposed to $w_{1}$.

As $\left(m_{1}, w_{1}\right)$ are no longer matched this implies that $w_{1}$ rejected $m_{1}$. Thus, we may conclude that $w_{1}$ prefers her current DA matching partner to $m_{1}$. This is a contradiction.

Another important property of the deferred acceptance algorithm is that each matching is man optimal. A stable match is considered man-optimal if every $\mathrm{m}$ in $\mathrm{M}$ prefers his partner to any partner he could possibly have in a stable matching. We define for each $m$, a woman $w$, as possible for $m$, if there is some stable outcome $x$ for which $x(m)=w$. For this proof, it is enough to prove that no $m$ in $\mathrm{M}$ is ever rejected by a $w$ in $\mathrm{W}$ who is possible for him. This is the case since we know that men propose in a decreasing order of preference (Lemma 3.1). Therefore, if a man is never rejected by a possible $w$ in W, we know that each $m$ is matched with their "best possible" match according to their preference list.

\section{Theorem 3.5. Upon termination each matching is man optimal.}

Proof. This will be proven with a proof by induction.

The base case $(n=1)$ : In round 1 of the DA algorithm, $m_{i}$ proposes to $w_{i}$ (where $w_{i}$ is the $\mathrm{w}$ in $\mathrm{W}$ that $m_{i}$ values the most). We distinguish between two cases:

Case 1: $w_{i}$ does not receive any other proposals this round.

As this is the first round of the algorithm, we know that $w_{i}$ has not received any other offers before, thus $w_{i}$ does not reject $m_{i}$ as this is her first proposal.

Case 2: $w_{i}$ receives other proposals this round.

Assume some $m_{j}$ proposes to $w_{i}$. We know that $w_{i}$ only accepts the proposal from $m_{j}$ (and rejects $m_{i}$ ) if she values $m_{j}$ over $m_{i}$, therefore in this case $\left(m_{i}, w_{i}\right)$ is not a stable match, thus $w_{i}$ is not a possible partner for $m_{i}$. Assume the induction hypothesis holds for round n: $m_{i}$ proposed to possible $w_{i}, m_{i}$ is not rejected. Prove for $(n+1)$ : Assume the opposite holds, at round $(n+1), w_{i}$ rejects $m_{i}$. Therefore, we know $m_{j}$ proposed to $w_{i}$ in round $n+1$. Thus, by the inductive hypothesis we know that all $w$ that $m_{j}$ proposed to in previous rounds have rejected $m_{j}$ and were impossible for $m_{j}$. We also know that $w_{i}$ prefers $m_{j}$ to $m_{i}$ (she rejects $\left.m_{i}\right)$. Thus, $\left(m_{i}, w_{i}\right)$ cannot be a stable match as otherwise $\left(m_{j}, w_{i}\right)$ would be a blocking pair. This is a contradiction.

Another important property of the deferred acceptance algorithm is that men have the dominant strategy to truthfully report their preferences. Let $\mathrm{P}$ be the preference profile where all $\mathrm{m}$ in $\mathrm{M}$ report their preferences truthfully. We define the following:

School Choice: An analysis of the strategic behaviour of students and schools in the student high-school allocation problem 
- $x=g(P)=$ the outcome of the preference profile P.

- $y=g\left(P^{\prime}\right)=$ the outcome of the preference profile $P^{\prime}$ which differs from $P$ with only one $m_{i}$ changing his true preferences, all other preferences are kept fixed.

- $G(P)=$ a specific realization of $\mathrm{P}$; we can refer to the $m$ rejected at a specific round of $\mathrm{G}(\mathrm{P})$.

By means of these definitions, to prove that men have the dominant strategy to truthfully report their preferences, it is sufficient to show: $g(P)>_{m_{i}} g\left(P^{\prime}\right)$. As this would imply that men would always prefer their truthful match over their match when misreporting their preferences. We only look at a subset of misrepresentations, namely the successful misrepresentations. We define a successful misrepresentation $P^{\prime}\left(m_{i}\right)$ by $m_{i}$ if $y\left(m_{i}\right) P\left(m_{i}\right) x\left(m_{i}\right)$ i.e. $m_{i}$ prefers $y\left(m_{i}\right)$ (the partner he's matched with when misrepresenting his preferences) over $x\left(m_{i}\right)$ (the partner he's matched with when reporting his preferences truthfully). Next, we define another type of misrepresentation $P^{\prime \prime}$ s.t. $y\left(m_{i}\right) P^{\prime \prime}\left(m_{i}\right) w_{j} \forall \mathrm{w}_{j} \neq \mathrm{y}\left(\mathrm{m}_{i}\right) . P^{\prime \prime}\left(m_{i}\right)$ is referred to as a simple misrepresentation. Before diving into the complete proof of truthful reporting being a dominant strategy for all $\mathrm{m}$ in $\mathrm{M}$, we first prove the following two lemmas.

Lemma 3.6. Let $y=g\left(P^{\prime}\right)$ and $z=g\left(P^{\prime \prime}\right)$, then $z\left(m_{i}\right)=y\left(m_{i}\right)$.

This lemma simply states that $m_{i}$ will end up being matched to the same partner $y\left(m_{i}\right)$ if he misrepresents his preferences using $P^{\prime}\left(m_{i}\right)$ or $P^{\prime \prime}\left(m_{i}\right)$.

Proof. We know that $\left(m_{i}, y\left(m_{i}\right)\right)$ is a stable match in $P^{\prime}$, since no new instabilities arise when switching to $\mathrm{P}$ " (simply $m_{i}$ proposes to $y\left(m_{i}\right)$ sooner), we may conclude that $y\left(m_{i}\right)$ is the best possible match for $m_{i}$ w.r.t. $P^{\prime \prime}$. As proved before we know that the Deferred Acceptance algorithm results in each $m$ being matched with their best possible match. Therefore, $z\left(m_{i}\right)=y\left(m_{i}\right)$.

By means of lemma 3.6 we now know that $m_{i}$ gets matched with the same $\mathrm{w}$ in both $P^{\prime}$ and $P^{\prime \prime}$, therefore to prove that truthful reporting is a dominant strategy we can now prove: $g(P)>_{m_{i}} g\left(P^{\prime \prime}\right)$.

Lemma 3.7. If $P^{\prime}\left(m_{i}\right)$ is a simple misrepresentation with $y=g\left(P^{\prime}\right)$ with either one of the following properties:

- $y\left(m_{i}\right) P\left(m_{i}\right) x\left(m_{i}\right)$ i.e. $m_{i}$ prefers $y\left(m_{i}\right)$ over their truthful match

- $y\left(m_{i}\right)=x\left(m_{i}\right)$

We have the for each $m_{j}$ in $M$ either one of the following properties holds:

- $y\left(m_{j}\right) P\left(m_{j}\right) x\left(m_{j}\right)$

- $y\left(m_{j}\right)=x\left(m_{j}\right)$

This lemma simply states that every $\mathrm{m}$ in M likes the outcome from the misrepresentation $y=g\left(P^{\prime}\right)$ at least as much as the outcome from truthfully reporting $x=g(P)$.

$84 \mid \begin{aligned} & \text { Marble } \\ & \text { Research } \\ & \text { Papers }\end{aligned}$


Proof. Assume the opposite holds, namely that $x\left(m_{j}\right) P\left(m_{j}\right) y\left(m_{j}\right)$ for some $m_{j}$ in $\mathrm{M}$. Thus, we assume that every $m$ in $\mathrm{M}$ other than $m_{i}$ prefer the outcome $\mathrm{x}$ over $\mathrm{y}$. We know that in $\mathrm{P}$ and $\mathrm{P}^{\prime}$ every $m$ in $\mathrm{M}$ (other than $m_{i}$ state the same preference profiles. Therefore, we know that $m_{j}$ is rejected by $x\left(m_{j}\right)$ in some round of $\mathrm{G}\left(\mathrm{P}^{\prime}\right)$. Let $n$ be the first round of $\mathrm{G}\left(\mathrm{P}^{\prime}\right)$ at which some $m_{j}$ is rejected by their $x\left(m_{j}\right)$. Then, as $x\left(m_{j}\right)$ is rejecting $m_{j}$ we know that:

a. In this same round $n, x\left(m_{j}\right)$ receives a proposal by some $m_{k}$ that did not propose to her in $\mathrm{G}(\mathrm{P})$.

b. $x\left(m_{j}\right)$ prefers $m_{k}$ over $m_{j}: m_{k} P\left(x\left(m_{j}\right)\right) m_{j}$.

From (a) we know that $x\left(m_{k}\right) P\left(m_{k}\right) x\left(m_{j}\right)$, so $m_{k}$ must have been rejected by $x\left(m_{k}\right)$ prior to step $\mathrm{n}$, which is a contradiction as we assumed step $\mathrm{n}$ to be the first round of such an instance. Therefore, no $m_{j}$ is rejected by their $x\left(m_{j}\right)$ in $G\left(P^{\prime}\right)$.

Our next step for proving that truthful reporting is a dominant strategy for all $\mathrm{m}$ in $\mathrm{M}$, is to show that $g(P)>_{m_{i}} g\left(P^{\prime \prime}\right)$, using the above two lemmas.

Theorem 3.8. $g(P)>_{m} g\left(P^{\prime \prime}\right)$ for all $m$ in $M$.

Proof. Let $\mathrm{P}$ be the preference profile where all $\mathrm{m}$ in $\mathrm{M}$ report truthfully, where $\mathrm{x}=\mathrm{g}(\mathrm{p})$ are the results of this preference profile. Let $l$ be the number of rounds it takes before the algorithm terminates given the preference profile $\mathrm{P}$. Let $\mathrm{P}^{\prime}$ be the preference profile where $m_{i}$ makes a simple misrepresentation and obtains $\mathrm{y}=\mathrm{g}\left(\mathrm{P}^{\prime}\right)$. We want to prove that the misrepresentation $\mathrm{P}^{\prime}$ cannot be successful. For any $m_{j}$ in $\mathrm{M}$, define making a final match in round $k$ of $\mathrm{G}(\mathrm{P})$ if $m_{j}$ proposes to his ultimate partner $x\left(m_{j}\right)$ in round $k$. Each $m_{j}$ in $\mathrm{M}$ can only make a match exactly once. We distinguish between two cases:

To show: $y\left(m_{j}\right)=x\left(m_{j}\right)$

Case 1: $m_{j}$ makes a final match at round $l$ of $\mathrm{P}$ (the final round of $\mathrm{P}$ ).

Since $l$ is the final round of $\mathrm{G}(\mathrm{P})$ and $m_{j}$ makes a final match with $x\left(m_{j}\right)$, we know that $m_{j}$ was the only man who proposed to $x\left(m_{j}\right)$ (since otherwise the algorithm would need another round before terminating). We know that no $\mathrm{m}$ proposes to $x\left(m_{j}\right)$ in $\mathrm{G}\left(\mathrm{P}^{\prime}\right)$ who didn't propose to $x\left(m_{j}\right)$ in $\mathrm{G}(\mathrm{P})$. Therefore, only $m_{j}$ proposes to $x\left(m_{j}\right)$ in $\mathrm{G}\left(\mathrm{P}^{\prime}\right)$ (since we know that $x\left(m_{j}\right)$ must receive at least one proposal). Thus we may conclude that $y\left(m_{j}\right)=x\left(m_{j}\right)$. Obviously, the same holds for any $m_{j}$ who is the only $\mathrm{m}$ who proposes to $x\left(m_{j}\right)$ in $\mathrm{G}(\mathrm{P})$, regardless of the round in which $m_{j}$ proposes to $x\left(m_{j}\right)$. Thus, if the manipulator $m_{i}$ either:

- Made a final match at round $l$ (the final round of $\mathrm{G}(\mathrm{P})$ ).

- Is the only $m$ who proposes to $x\left(m_{i}\right)$.

We know that $y\left(m_{i}\right)=x\left(m_{i}\right)$, and therefore we don't have $y\left(m_{i}\right) P\left(m_{i}\right) x\left(m_{i}\right)$ and his manipulation cannot be successful.

Case 2: $m_{i}$ makes a final match at some round $k$ in $\mathrm{G}(\mathrm{P})$ s.t. $1 \leq k<l$.

To show: For every $\mathrm{m}$ in $\mathrm{M}$ (including the manipulator $m_{i}$ ) who makes a final match at step $k$ or later, we have $y(m)=x(m)$.

Let $r$ be a round in $\mathrm{G}(\mathrm{P})$ s.t. $k \leq r<l$. We have already shown that $y\left(m_{j}\right)=x\left(m_{j}\right)$

School Choice: An analysis of the strategic behaviour of students and schools in the student high-school allocation problem 
holds for the final round $l$ of $\mathrm{G}(\mathrm{P})$.

Assume that the inductive hypothesis $y\left(m_{j}\right)=x\left(m_{j}\right)$ holds for round $\mathrm{r}$.

Prove for round $r+1$ : Let $m_{q}$ be an $\mathrm{m}$ in $\mathrm{M}$ that makes a final match at round $\mathrm{r}$ of $\mathrm{G}(\mathrm{P})$. Let $\mathrm{M}$ ' be the subset of $m_{j}$ in $\mathrm{M}$ who were rejected by $x\left(m_{q}\right)$ in $\mathrm{G}(\mathrm{P})$ i.e. $M^{\prime}=\left[m_{j} \in M \mid x\left(m_{q}\right) P\left(m_{j}\right) x\left(m_{j}\right)\right]$. Thus, $M^{\prime}$ is the subset of $m$ in M that prefer $x\left(m_{q}\right)$ over their final partner $x\left(m_{j}\right)$. If $M^{\prime}$ is empty, we know that $m_{q}$ is the only $\mathrm{m}$ in $\mathrm{M}$ who proposes to $x\left(m_{q}\right)$ and by caes 1 we may conclude that $y\left(m_{q}\right)=x\left(m_{q}\right)$.

Assume $M^{\prime}$ is non-empty, let $m_{u}$ be in $M^{\prime}$ s.t. $m_{u} P\left(x\left(m_{q}\right)\right) m_{s}$ for all other men $m_{s}$ in $M^{\prime}$. That is, $m_{u}$ is preferred by $x\left(m_{q}\right)$ over all other men she rejected in $\mathrm{G}(\mathrm{P})$. Then, we know that $m_{u}$ makes his final match after round $r$ of $\mathrm{G}(\mathrm{P})$, since he's not rejected by $x\left(m_{q}\right)$ until round $r$. Therefore, by the induction hypothesis we know that $y\left(m_{u}\right)=x\left(m_{u}\right)$. As we know that $m_{u}$ is not the manipulator, this means that $m_{u}$ proposes to $x\left(m_{q}\right)$ in $\mathrm{G}\left(\mathrm{P}^{\prime}\right)$ and is rejected in favor of someone who $x\left(m_{q}\right)$ prefers. But since no $m$ proposes to a $w$ in $P^{\prime}$ if he didn't already propose to her in $P$, we know that $x\left(m_{q}\right)$ rejects $m_{u}$ for $m_{q}$, therefore we have $y\left(m_{u}\right)=x\left(m_{u}\right)$.

By induction we have that $y\left(m_{j}\right)=x\left(m_{j}\right)$ for every $m_{j}$ who makes a match at step $k$ or later. Including $m_{i}$, therefore we may conclude that the manipulation is not successful. Thus, all men have the dominant strategy to truthfull report their preferences. Although the deferred acceptance algorithm results in the man-optimal stable match, it is important to note that the resulting match may not be Pareto efficient for the men. This is since the algorithm also take the women's preferences into consideration.

Another important property of the deferred acceptance algorithm is that some $w$ in $\mathrm{W}$ can manipulate the outcome by not reporting truthfully. Pini, Rossi and Venable (2009) provide the conditions under which manipulation is possible, given a preference profile $\mathrm{p}$, some women $w, v$ in $\mathrm{W}$; and some men $m, n$ in M. Firstly, let $m=$ the male optimal partner for $w$ in $\mathrm{p}$. Let $n=$ the female optimal partner for $w$ in $\mathrm{p}$. Let $n=$ the male optimal partner for $v$ in $\mathrm{p}$.

The preference profile $\mathrm{p}$ is manipulable by $w$ if and only if:

- $w$ receives more than 1 proposal.

- $v$ prefers $m$ to $n$

- $n$ prefers $v$ to $w$

- $m$ prefers $w$ to $v$

Based on these conditions we construct the following 3x3 example of a preference profile in which a woman can manipulate the outcome of the deferred acceptance algorithm (Table 3 and table 4).

\begin{tabular}{|c|ccc|}
\hline$n$ & $v$ & $w$ & $u$ \\
$m$ & $w$ & $v$ & $u$ \\
$o$ & $w$ & $u$ & $v$ \\
\hline
\end{tabular}

Table 3: Male preferences

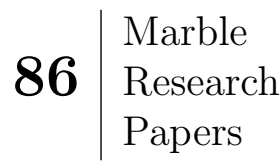




\begin{tabular}{|c|ccc|}
\hline$w$ & $n$ & $m$ & $o$ \\
$v$ & $m$ & $n$ & $o$ \\
$u$ & $n$ & $o$ & $m$ \\
\hline
\end{tabular}

Table 4: Female preferences

Example 3.2. Given the preference profiles in Table 3 and 4 the DA algorithm results in the matching: $[(n, v) ;(m, w) ;(o, u)]$. w can manipulate the outcome by changing her reported preference, this gives us the updated preference profile as noted in Table 5.

\begin{tabular}{|c|ccc|}
\hline$w$ & $n$ & $o$ & $m$ \\
$v$ & $m$ & $n$ & $o$ \\
$u$ & $n$ & $o$ & $m$ \\
\hline
\end{tabular}

Table 5: Updated female preferences

Given these updated preference profiles the DA algorithm results in the following maleoptimal pairs $[(n, w) ;(m, v) ;(o, u)]$. In these new matching pairs both $w$ and $v$ improve by being matched with a male they truthfully value higher than their initial partner.

Example 3.2 shows that for the deferred acceptance algorithm it is not a dominant strategy for women to report truthfully.

\section{Extending to School Choice}

In this section the Deferred Acceptance algorithm will be extended to the school choice problem. Firstly, we will introduce the school choice model. Following that, the model will be adjusted to fit the Deferred Acceptance algorithm. The main properties of the new model will be discussed. Finally, we consider the manipulability of the algorithm.

\subsection{The School Choice Model}

Further applications of the deferred acceptance algorithm include many-to-one matching problems. The main focus of this paper is the problem of assigning students to highschools. In this case, we will look at the student-proposing algorithm, i.e. the students will replace the men, and the high-schools will represent the women. The many-to-one school choice model looks as follows:

- Set of schools $\mathrm{S}$, each $s_{i}$ in $\mathrm{S}$ has a capacity $q_{i}$

- Set of students L

- Set of preferences $\mathrm{P}$ (of each $l$ in L)

- Set of school preferences/priorities $\pi$

School Choice: An analysis of the strategic behaviour of students and schools in the student high-school allocation problem 
We distinguish between two cases of the school choice problem

- All schools rank students with the same criteria, resulting in all $s$ in $\mathrm{S}$ having the same preferences over the students.

- Some schools use their own individual criteria for ranking students, thus the preferences of the schools differ.

In this paper we look at the case where schools use different criteria for ranking students.

\subsection{Adjusting the School Choice Model}

Unlike the initial marriage problem, schools accept multiple students. A matching in the school choice problem is an outcome where each student is matched to at most one school, and each school is matched to at most its capacity $q$ of students. However, in this model schools only provide their preferences over the students, not over the outcome of the group of students. It is impossible to determine whether a school with a capacity of 3 would prefer the outcome $\left(l_{1}, l_{6}, l_{7}\right)$ or $\left(l_{3}, l_{4}, l_{5}\right)$ where $l_{i}$ indicates the student ranked at the $i t h$ spot. Mathematically this implies that for a given school $s_{i}$ with $q_{i}$ spots, for any subset $L^{\prime}$ of students s.t. $|L|<q_{i}$ and $l_{i}, l_{j} \notin L$, we know the following:

- $s_{i}$ prefers $l_{i} \cup L^{\prime}$ to $l_{j} \cup L^{\prime}$ i.f.f. $s_{i}$ prefers $l_{i}$ to $l_{j}$

- $s_{i}$ prefers $l_{i} \cup L^{\prime}$ to $L^{\prime}$ i.f.f. $\left|L^{\prime}\right|<q_{i}$

This implies that for each $s_{i}$ in $\mathrm{S}$ there is no student who is only/more desirable if some other student is also going to that school $s_{i}$. That is, in this model students are not compliments but rather substitutes. This allows us to be able to transform the many-toone school choice problem to a one-to-one matching problem by doing the following:

- Replace school $s_{i}$ by $q_{i}$ copies of $s_{i}$. Each copy of $s_{i}$ has the same preference list $\pi_{i}$

- Modify each student's preference list by replacing $s_{i}$ by the array $s_{i 1}, s_{i 2}, \ldots, s_{i q_{i}}$

Following that, a matching $V$ of the many-to-one school choice problem is equivalent to the matching $V^{\prime}$ in this modified one-to-one model. Therefore, the properties of the Deferred Acceptance algorithm matching discussed in section 2 hold for $V^{\prime}$. Thus, showing that the following properties hold for $V$ is trivial:

- $\mathrm{V}$ is stable.

- All students in $\mathrm{V}$ are matched.

- It is a dominant strategy for students to truthfully report their preferences.

$88 \mid \begin{aligned} & \text { Marble } \\ & \text { Research } \\ & \text { Papers }\end{aligned}$




\subsection{Manipulation}

Given the aforementioned method it is obvious that the conditions for manipulability in the school problem will be similar to those proposed by Pini, Rossi and Venable (2009) for solving the marriage problem. We will derive these conditions by constructing the smallest possible example where manipulation is possible for some school w with $q_{w}$. We must have that for at least one school s, $q_{s}>1$ (otherwise the school problem can be solved as a marriage problem without making any adjustments). Thus, let $q_{w}=2$ and $q_{v}=1$. Let there only be two different preference profiles. Consequently we define given a preference profile $\mathrm{p}$ (which results in the match $\mu$ ), some schools $\mathrm{w}, \mathrm{v}$ with $q_{w}, q_{v}$; and some students $\mathrm{m}, \mathrm{n}$ and $\mathrm{o}$ the following:

- $m \in \mu(w)$ in the student proposing version of the algorithm for $\mathrm{p}$

- $n \in \mu(w)$ in the schools proposing version of the algorithm for $\mathrm{p}$

- $n \in \mu(v)$ in the student proposing version of the algorithm for $\mathrm{p}$

Furthermore, given the Pini, Rossi and Venable (2009) conditions we need the following conditions for $\mathrm{p}$ :

- $\mathrm{w}$ receives more than 1 application.

- $\mathrm{v}$ prefers $m$ to $\mathrm{n}$.

- $\mathrm{n}$ prefers $\mathrm{v}$ to $\mathrm{w}$.

- $\mathrm{m}$ prefers $\mathrm{w}$ to $\mathrm{v}$.

The only $3 \times 3$ preferences in which the aforementioned conditions hold, is the following (see Table 6 and table 7 ):

\begin{tabular}{|c|ccc|}
\hline $\mathrm{m}$ & $w_{1}$ & $w_{2}$ & $\mathrm{v}$ \\
$\mathrm{n}$ & $\mathrm{v}$ & $w_{1}$ & $w_{2}$ \\
$\mathrm{o}$ & $w_{1}$ & $w_{2}$ & $\mathrm{v}$ \\
\hline
\end{tabular}

Table 6: Student preferences

\begin{tabular}{|c|ccc|}
\hline$w_{1}$ & $\mathrm{~m}$ & $\mathrm{n}$ & $\mathrm{o}$ \\
$w_{2}$ & $\mathrm{~m}$ & $\mathrm{n}$ & $\mathrm{o}$ \\
$\mathrm{v}$ & $\mathrm{m}$ & $\mathrm{o}$ & $\mathrm{n}$ \\
\hline
\end{tabular}

Table 7: School preferences

Given these preference profiles, the Deferred Acceptance algorithm results in the stable matching: $[(w, m, o) ;(v, n)]$. A successful manipulation for w would be one where they end up with the following stable matching: $[(w, m, n) ;(v, o)]$. By checking for all 5 possibilities where $\mathrm{w}$ misreports their preferences, we conclude that in this preference setting manipulation is not possible. In each manipulation case, the algorithm terminates after

School Choice: An analysis of the strategic behaviour of students and schools in the student high-school allocation problem 
one round; we note that the condition that "w receives more than 1 application" is not strong enough, as $q_{w}=2$. We update our conditions accordingly and construct a $4 \times 4$ preference profile where the following holds:

- w receives more than $q_{w}$ proposals.

- $\mathrm{v}$ prefers $\mathrm{m}$ to $\mathrm{n}$.

- $\mathrm{n}$ prefers $\mathrm{v}$ to $\mathrm{w}$.

- $\mathrm{m}$ prefers $\mathrm{w}$ to $\mathrm{v}$.

Given these conditions we construct a $4 \times 4$ in Example 4.1 where a school is able to manipulate by misrepresenting their preferences.

Example 4.1. We have 3 schools: $w, v$ and $u$ with the following quantities $q_{w}=2, q_{v}=1$ and $q_{u}=1$. We have 4 students: $n, m, o$ and $p$. To be able to solve this many-to-one school problem we first adjust the problem to the one-to-one model. This results in the following one-to-one preference profiles (Table 8 and table 9):

\begin{tabular}{|c|cccc|}
\hline $\mathrm{n}$ & $\mathrm{v}$ & $w_{1}$ & $w_{2}$ & $\mathrm{u}$ \\
$\mathrm{m}$ & $w_{1}$ & $w_{2}$ & $\mathrm{v}$ & $\mathrm{u}$ \\
$\mathrm{o}$ & $w_{1}$ & $w_{2}$ & $\mathrm{u}$ & $\mathrm{v}$ \\
$\mathrm{p}$ & $w_{1}$ & $w_{2}$ & $\mathrm{u}$ & $\mathrm{v}$ \\
\hline
\end{tabular}

Table 8: Student preferences

\begin{tabular}{|c|cccc|}
\hline$w_{1}$ & $\mathrm{n}$ & $\mathrm{o}$ & $\mathrm{m}$ & $\mathrm{p}$ \\
$w_{2}$ & $\mathrm{n}$ & $\mathrm{o}$ & $\mathrm{m}$ & $\mathrm{p}$ \\
$\mathrm{v}$ & $\mathrm{m}$ & $\mathrm{n}$ & $\mathrm{o}$ & $\mathrm{p}$ \\
$\mathrm{u}$ & $\mathrm{m}$ & $\mathrm{n}$ & $\mathrm{o}$ & $\mathrm{p}$ \\
\hline
\end{tabular}

Table 9: School preferences

Given these preference profiles the Deferred Acceptance algorithm results in the following stable matching: $[(w, m, o) ;(v, n) ;(u, p)]$. School $w$ is able to manipulate the matching by updating his preferences to the following (Table 10):

\begin{tabular}{|c|cccc|}
\hline$w_{1}$ & $\mathrm{n}$ & $\mathrm{o}$ & $\mathrm{p}$ & $\mathrm{m}$ \\
$w_{2}$ & $\mathrm{n}$ & $\mathrm{o}$ & $\mathrm{p}$ & $\mathrm{m}$ \\
$\mathrm{v}$ & $\mathrm{m}$ & $\mathrm{n}$ & $\mathrm{o}$ & $\mathrm{p}$ \\
$\mathrm{u}$ & $\mathrm{m}$ & $\mathrm{n}$ & $\mathrm{o}$ & $\mathrm{p}$ \\
\hline
\end{tabular}

Table 10: Updated school preferences (with w misreporting)

Given this updated preference profile the Deferred Acceptance algorithm results in the following stable matching: $[(w, n, o) ;(v, m) ;(u, p)]$. In this case we have that by the misreporting of school $w$, both schools $v$ and $w$ are able to improve their matching. Therefore it is possible for schools to behave strategically like a coalition to improve their matching.

$90 \mid \begin{aligned} & \text { Marble } \\ & \text { Research } \\ & \text { Papers }\end{aligned}$ 
Example 4.1 shows that the aforementioned conditions are enough for a school w to be able to successfully manipulate.

\section{Conclusion}

\subsection{Summary}

In this paper we analyzed the strategic behavior of students and schools in the Deferred Acceptance algorithm. We showed that the Deferred Acceptance algorithm results in a matching that is both complete and stable. Furthermore, we found that students have the dominant strategy to truthfully report their preferences. We assessed the cases with three schools with two preferences and four students to construct the conditions under which schools are able to manipulate. Given a preference profile $\mathrm{p}$ (which results in the match $\mu$ ), some schools $\mathrm{w}, \mathrm{v}$ with $q_{w}, q_{v}$; and some students $\mathrm{m}, \mathrm{n}$ and $\mathrm{o}$; we find that the following conditions are sufficient to construct a manipulable example:

- $m \in \mu(w)$ in the student proposing version of the algorithm for $\mathrm{p}$

- $n \in \mu(w)$ in the schools proposing version of the algorithm for $\mathrm{p}$

- $n \in \mu(v)$ in the student proposing version of the algorithm for $\mathrm{p}$

- $\mathrm{w}$ receives more than $q_{w}$ proposals.

- $\mathrm{v}$ prefers $\mathrm{m}$ to $\mathrm{n}$.

- $\mathrm{n}$ prefers $\mathrm{v}$ to $\mathrm{w}$.

- $\mathrm{m}$ prefers $\mathrm{w}$ to $\mathrm{v}$.

\subsection{Implications}

The results provide important insight into the behavior of students and schools in this model. Due to truthful reporting being a dominant strategy for students, we know that students do not have an incentive to cheat by misrepresenting their preferences. Furthermore, by keeping the ability to manipulate on the side of the schools the model can be controlled. We would like to emphasize that the given conditions under which schools can manipulate are rather explicit. Thus, the amount cases in which a school would actually be able to successfully manipulate are small.

\subsection{Improvements}

There are some extensions that we could propose. Firstly, one could consider the cases where all schools have the same preferences. This would be the case if one central exam would be the determinant for all schools. Furthermore, one could consider a model where schools do have individual preferences over groups of students.

School Choice: An analysis of the strategic behaviour of students and schools in the student high-school allocation problem 


\section{References}

[1] Paul Milgrom and Ilya Segal (2015) Deferred-acceptance auctions and radio spectrum reallocation.

[2] Kojima, F., Ünver, M. U. (2013) The "Boston" school-choice mechanism: an axiomatic approach. Economic Theory, 55(3).

[3] Atila Abdulkadiroğlu and Tayfun Sönmez (2013) School Choice: A Mechanism Design Approach. American Economic Review 93-3: 729-747.

[4] M.S. Pini, F. Rossi, K.B. Venable, T. Walsh (2009) Manipulation and gender neutrality in stable marriage procedures. Proc. of 8th Int. Conf. on Autonomous Agents and Multiagent Systems.

Marble

92 Research

Papers 